\title{
A saúde sexual de usuários de aplicativos de relacionamento
}

\author{
The sexual health of users of dating apps \\ La salud sexual de usuarios de aplicaciones de citas \\ Bruna Benício Rodrigues ${ }^{1 *}$, Sandro Caramaschi ${ }^{1}$.
}

\begin{abstract}
RESUMO
Objetivo: Verificar como os usuários de aplicativos de relacionamento exercem sua sexualidade com os parceiros virtuais e realizar uma comparação entre o sexo feminino e masculino. Métodos: Trata-se de uma pesquisa de campo, realizada com 210 indivíduos, de ambos os sexos, com idade média de 21,35 anos. Os participantes responderam a um questionário presencialmente que continha o Inventário de Orientação Sociossexual e duas questões elaboradas a respeito do seu exercício sexual com parceiros virtuais. Os dados foram submetidos à análise estatística descritiva e ao Teste t de Student para a comparação entre os sexos. O estudo foi aprovado por Comitê de Ética em Pesquisa. Resultados: Os resultados demonstraram que perante o uso de aplicativos de relacionamento, os homens estão mais dispostos a manterem relações sexuais independente das circunstâncias quando comparados às mulheres. Conclusão: Foi possível concluir que diante dos resultados levantados é necessário a elaboração de propostas de políticas públicas aplicada à população para proporcionar uma educação sexual aos usuários dos aplicativos de relacionamento e cidadãos em geral, para haver a prevenção de quadros indesejáveis como a aquisição de doenças sexualmente transmissíveis ou gravidez indesejada.
\end{abstract}

Palavras-chave: Sexualidade, Prevenção, Educação sexual.

\begin{abstract}
Objective: To verify how users of relationship applications exercise their sexuality with virtual partners and to compare women and men. Methods: This is a field research, does with 210 individuals, of two sexes, with an average age of 21.35 years. The participants answered a questionnaire in person that contained the Inventory of Sociosexual Orientation and two questions elaborated about their sexual exercise with virtual partners. The data were submitted to descriptive statistical analysis and Student's t-test for comparison between genders. The study was approved by the Research Ethics Committee. Results: The results showed that when using relationship apps, men are more willing to have sex regardless of circumstances when compared to women. Conclusion: It was possible to conclude that in view of the results obtained, it is necessary to elaborate public policy proposals applied to the population to provide sexual education to users of relationship applications and citizens in general, in order to prevent undesirable conditions such as the acquisition of diseases sexually transmitted or unwanted pregnancy.
\end{abstract}

Keywords: Sexuality, Prevention, Sex education.

RESUMEN

Objetivo: verificar cómo los usuarios de aplicaciones de relaciones ejercen su sexualidad con parejas virtuales y comparar mujeres y hombres. Métodos: Esta es una investigación de campo, realizada con 210

1 Universidade Estadual Paulista "Júlio de Mesquita Filho" (UNESP), Bauru - SP.

*E-mail: bru_benicio@hotmail.com 
individuos, de ambos sexos, con una edad promedio de 21.35 años. Los participantes respondieron un cuestionario en persona que contenía el Inventario de Orientación Sociosexual y dos preguntas elaboradas sobre su ejercicio sexual con parejas virtuales. Los datos se sometieron a un análisis estadístico descriptivo y a la prueba t de Student para la comparación entre géneros. El estudio fue aprobado por el Comité de Ética en Investigación. Resultados: Los resultados mostraron que cuando se usan aplicaciones de relación, los hombres están más dispuestos a tener relaciones sexuales independientemente de las circunstancias en comparación con las mujeres. Conclusión: fue posible concluir que, en vista de los resultados obtenidos, es necesario elaborar propuestas de políticas públicas aplicadas a la población para proporcionar educación sexual a los usuarios de las aplicaciones de relación y a los ciudadanos en general, a fin de prevenir condiciones no deseadas como la adquisición de enfermedades y embarazo de transmisión sexual o no deseado.

Palabras clave: Sexualidad, Prevención, Educación sexual.

\section{INTRODUÇÃO}

A preferência sexual que distingue homens e mulheres sofrem influências genéticas e biológicas, além de fatores culturais. Com isso, as características desejadas e a quantidade de parceiros sexuais diferenciou-se entre os sexos conforme apontou Hattori WT e Castro FN (2017). Polippo PM, et al. (2016) alegam que os estudos brasileiros negligenciam as predisposições genéticas que se manifestam durante a seleção de parceiros e portanto, afirmam a necessidade de aumentar sua produção científica.

Os autores explicam que ao decorrer da evolução humana, o cérebro se modificou e isso resultou em diferenciações na estrutura feminina e masculina. Assim, os comportamentos que proporcionaram a melhor adaptação ao ambiente a depender do sexo biológico foram armazenados na mente humana e repassados às gerações seguintes.

Trivers RL (1972) ao observar que a maturação do bebê humano é demorada em relação aos demais animais, criou a chamada Teoria do Investimento Parental em que propõe que visando a sobrevivência da cria, ambos os sexos diferenciaram suas preferências durante a escolha parental.

As mulheres por ficarem em estado físico debilitado durante a gestação e lactação, desenvolveram o interesse por se relacionarem com parceiros que seriam considerados capazes de prover alimento e segurança para ela e a prole. Do mesmo modo, passaram a se interessar por relações sexuais restritas, uma vez que a fecundação seria possível a partir da relação sexual com um único parceiro.

Os homens, por sua vez, passaram a avaliar as parceiras por meio das características físicas, já que os níveis hormonais equilibrados eram demonstrados por meio da estética e estavam intimamente relacionados à capacidade reprodutiva e gestação saudável.

Diante disso, passaram a se interessar por um envolvimento sexual com diferentes parceiras já que era possível aumentar a possibilidade de fecundação ao se relacionar sexualmente com a maior quantidade de parceiras que conseguirem e assim, contribuirem para a propagação e aumento da espécie (HATTORI WT e CASTRO FN, 2017; BANDEIRA TTA e MOURA MLS, 2012; PINCOTT J, 2010).

Entretanto, atualmente as relações amorosas sofreram os impactos da tecnologia e globalização, e com isso, os dados do Cetic.br (2017), Nogueira MFM e Silva TT (2016) e Santaella L (2010) apontaram que os aplicativos de relacionamento surgiram em função das mudanças globais e hoje o seu uso se tornou habitual e pertencente ao cotidiano dos indivíduos. Rocha CTM e Amador FS (2018) mencionam que perante o grande número de atividades exigidas contemporaneamente, a busca por um parceiro romântico foi facilitada pelo surgimento de aplicativos destinados exclusivamente a essa finalidade.

Perante isso, Nogueira MFM e Silva TT (2016) apontam que a comunicação e as relações em geral não ocorrem mais somente em contextos presenciais mas agora contemplam os espaços virtuais. Ao realizarem pesquisas sobre o assunto, Hattori WT e Castro FN (2017) e Ramos CM e Lencastre MPA (2013) descobriram 
que a Teoria do Investimento Parental, mencionada acima, está presente na seleção de parceiros por meio de aplicativos de relacionamento, uma vez que os homens preferiam candidatas que demonstrassem beleza física por meio das fotos.

Ramirez A, et al. (2014) ao verificarem qual era a dinâmica dos relacionamentos virtuais que obtiveram sucesso, constataram que aqueles que tiveram curta interação virtual seguida de um encontro presencial, inúmeras fotos pessoais disponíveis e mantiveram contato por diferentes meios virtuais como Facebook, WhatsApp ou Instagram, foram os que mais declararam satisfação com o parceiro. Isso ocorre, porque mediante a breve interação virtual, o sujeito não possui muito tempo para fantasiar aspectos relativos ao pretendente.

Figueiredo LB (2016) observou que mulheres que utilizaram o aplicativo Tinder, ainda que morassem sozinhas e que fossem independentes financeiramente, relatavam insatisfação consigo mesmas caso viessem se envolver em uma relação casual ou puramente sexual. Por outro lado, Sümer ZHL (2013) destaca que os homens e mulheres de sua pesquisa não diferiram em relação a manifestação sexual, o que propõe que a amostra dos estudos possibilita a variabilidade dos resultados.

Sawyer NA, et al. (2018) e Green SM, et al. (2018) apontaram que de um modo geral, os usuários de aplicativos de relacionamento possuem um número de parceiros sexuais maior do que os que não utilizam e estão envolvidos em práticas de sexo casual perigosas e relacionadas à contração de doenças sexualmente transmissíveis e vinculada ao abuso de álcool e drogas.

O uso de preservativos também foi negligenciado durante os encontros presenciais. A média de relação sexual com pretendentes virtuais foi de 30 parceiros variados e esses dados abrangem $96,5 \%$ dos usuários, sendo que apenas 3,5\% não estavam envolvidos em práticas sexuais de risco.

Diante disso, Overbeek G, et al. (2018) verificaram que as mulheres se envolvem em práticas sexuais menos perigosas do que os homens e possuem um número menor de parceiros sexuais por prezarem ter um envolvimento emocional antes do sexual. No entanto, Vermelho SC et al. (2015) a partir de uma revisão de literatura verificou que no Brasil pouco se sabe sobre os desdobramentos que ocorrem com o uso dos aplicativos de relacionamento devido ao baixo número de pesquisas que envolvem mídias digitais em contexto nacional.

Diante disso, a presente pesquisa teve o objetivo de apurar como os usuários brasileiros dos aplicativos de relacionamento desempenham suas atividades sexuais, realizando uma comparação estatística entre homens e mulheres.

\section{MÉTODOS}

Este trabalho contemplou uma pesquisa de campo, a partir da aplicação de um questionário em universitários matriculados em diferentes cursos de uma universidade estadual do interior do estado de São Paulo. Todos os procedimentos éticos previstos para a pesquisa com seres humanos foram seguidos e 0 projeto de pesquisa primeiramente foi enviado para avaliação do Comitê de Ética em Pesquisa da Universidade Estadual Paulista "Júlio de Mesquita Filho", Bauru-SP e após a aprovação (CAAE: 68504617.2.00005398; Número do Parecer: 2.100.031), iniciou-se a coleta

\section{Participantes}

Participaram da pesquisa aqueles que concordaram com o Termo de Consentimento Livre e Esclarecido, de modo voluntário e anônimo, resultando um total de 210 sujeitos, 101 do sexo masculino com idade média de 21,35 anos e 109 do feminino com idade média de 20,58 anos, matriculados em diferentes anos nos cursos de Psicologia, Jornalismo, Educação Física e Engenharia de Produção.

\section{Instrumentos}

Os dados apresentados nesse artigo foram coletados na pesquisa de mestrado acadêmico da autora, realizado em uma universidade pública localizada no interior do estado de São Paulo, no entanto, para sua 
interpretação foi realizada uma nova leitura dos dados. O método e os resultados presentes nesse artigo são semelhantes aos encontrados no banco de dados em que foi depositada a dissertação. Os resultados apresentados aqui representam apenas uma parcela dos dados encontrados na dissertação.

Foram utilizados como instrumentos para a coleta de dados o Inventário de Orientação Sociossexual (IOS) desenvolvido por Simpson JA e Gangestad SW (1991) que contempla sete questões em que o valor numérico de algumas deve ser invertido para ser pontuado, de modo a avaliar o comportamento e frequência sexual e assim, os que pontuarem acima da média condizente ao seu sexo $(F=82,47 ; M=112,87)$ são considerados mais irrestritos sexualmente e os que pontuarem abaixo da média, mais restritos sexualmente. Portanto, avalia a propensão do sujeito se envolver em práticas sexuais.

Por último, foi elaborado um questionário com perguntas de múltipla escolha e com escala, contendo informações pessoais e de seu próprio uso nos aplicativos de relacionamento.

Quadro 1 - Questões formuladas utilizadas nesse artigo.

\begin{tabular}{|c|}
\hline Atualmente você está amando alguém? \\
\hline $\begin{array}{l}\text { ( ) Não estou } \\
\text { ( ) Sim, há bastante tempo (anos) } \\
\text { ( ) Sim, há pouco tempo (meses) } \\
\text { ( ) Deixei de amar há pouco tempo (meses) } \\
\text { ( ) Deixei de amar há muito tempo (anos) }\end{array}$ \\
\hline Se respondeu sim à pergunta anterior, onde conheceu o seu parceiro(a)? \\
\hline $\begin{array}{l}\text { ( ) Na escola/faculdade } \\
\text { ( ) Em um bar/balada } \\
\text { ( ) Através de um amigo em comum } \\
\text { ( ) Via internet. Aplicativo(s) } \\
\text { Outros: }\end{array}$ \\
\hline Curto prazo(1) ou longo prazo(2) - Tabela 1: \\
\hline $\begin{array}{l}\text { 1) Eu não me importo de ter relação sexual no primeiro encontro com um(a) parceiro(a) que conheci por } \\
\text { meio da internet mesmo que ele(a) deixe claro que será algo casual e não há possibilidade de nos vermos } \\
\text { novamente } \\
\text { Discordo } 1 \ldots 2 \ldots 3 \ldots 4 \ldots 5 \ldots 6 \ldots 7 \ldots 8 \ldots 9 \ldots 10 \text { Concordo }\end{array}$ \\
\hline $\begin{array}{l}\text { 2) Por mim tudo bem ter relação sexual no primeiro encontro com um(a) parceiro(a) que conheci por meio } \\
\text { da internet desde que ao menos fique em aberto o futuro da relação, ou seja, não esteja combinado } \\
\text { necessariamente que irá se restringir a apenas uma noite. }\end{array}$ \\
\hline Discordo $1 \ldots 2 \ldots 3 \ldots 4 \ldots 5 \ldots 6 \ldots 7 \ldots 8 \ldots 9 \ldots 10$ Conc \\
\hline
\end{tabular}

Fonte: Rodrigues BB e Caramaschi S, 2019.

\section{Procedimentos de coleta}

Antes de responder ao questionário os participantes assinaram o Termo de Consentimento Livre e Esclarecido. O questionário foi aplicado coletivamente em salas de aula sendo respondido individualmente, com aprovação anterior do docente responsável pela disciplina, resguardando-se a ética e privacidade, com uma duração de aproximadamente 60 minutos.

\section{Análise dos dados}

As questões do questionário elaboradas foram analisadas quantitativamente e o inventário foi submetido à análise estatística descritiva (média e desvio-padrão), bem como realizado a comparação entre o sexo feminino e masculino (Teste T de Student). 


\section{RESULTADOS}

Participaram da pesquisa 210 sujeitos, sendo 101 do sexo masculino com idade média de 21,35 anos (com uma variabilidade de $\pm 2,92$ anos) e 109 do feminino com idade média de 20,58 anos (com uma variabilidade de $\pm 2,09$ ).

Em relação à amostra masculina, $4 \%$ conheceu a atual parceira com quem mantém uma relação duradoura pela internet e em relação à amostra feminina $8,2 \%$ conheceram o parceiro por meios virtuais. De acordo com a Tabela 1, podem-se observar os resultados estatísticos do Inventário de Orientação Sociossexual (IOS) e de duas questões elaboradas pelos pesquisadores (Curto Prazo e Longo Prazo).

De acordo com o Inventário de Orientação Sociossexual (IOS) constatou-se que os homens $(M=112,87$; $\mathrm{DP}=112,95)$ são mais irrestritos sexualmente e propensos a praticarem sexo casual do que as mulheres $(\mathrm{M}=82,47 ; \mathrm{DP}=61,03)$. De acordo com as duas questões elaboradas (Curto Prazo e Longo Prazo) os homens $(M=7.86$; $D P=2,88)$ pontuaram mais do que as mulheres $(M=5,86$; $D P=3,62)$ sobre a probabilidade de ter relação sexual no primeiro encontro $\left(t=-4,4414 ; p<0,0001^{*}\right)$, em situações que se restringiriam a uma relação casual.

Os homens $(M=5,15 ; D P=3,16)$ também pontuaram mais do que as mulheres $(M=4,09 ; D P=3,02)$ sobre a possibilidade de ter relação sexual no primeiro encontro em situações que propõe relacionamentos duradouros $\left(t=-2,4751 ; p=0,0145^{\star}\right)$.

Tabela 1 - Comparação estatística entre homens e mulheres (Teste t) relativa aos resultados do Inventário de Sociossexualidade (SOI) e de duas questões elaboradas.

\begin{tabular}{|c|c|c|c|c|c|c|}
\hline \multirow{2}{*}{$\begin{array}{c}\text { Participante } \\
\text { Variável }\end{array}$} & \multicolumn{2}{|c|}{ Feminino } & \multicolumn{2}{|c|}{ Masculino } & \multicolumn{2}{|c|}{ Estatística } \\
\hline & $\mathbf{M}$ & DP & $\mathbf{M}$ & DP & $T$ & $P$ \\
\hline SOI & 82,47 & 61,03 & 112,87 & 112,95 & 2,4000 & $0,0179^{*}$ \\
\hline Curto Prazo & 5,86 & 3,62 & 7,86 & 2,88 & $\begin{array}{c}- \\
4,4414\end{array}$ & $<0,0001^{*}$ \\
\hline Longo Prazo & 4,09 & 3,02 & 5,15 & 3,16 & $\begin{array}{c}- \\
2,4751\end{array}$ & $0,0145^{*}$ \\
\hline
\end{tabular}

Legenda: *significativo para $p<0,05$. Fonte: Rodrigues BB e Caramaschi S, 2019.

\section{DISCUSSÃO}

Com os dados gerais sobre os participantes foi possível constatar que a maioria deles não conheceu o atual parceiro por meio de um aplicativo de relacionamento, entretanto, outros dados presentes na dissertação demonstraram que os participantes, em sua maioria, usam ou já utilizaram em algum momento da vida a internet para selecionarem parceiros mas as relações resultaram em casualidade.

O dado obtido por meio do Inventário de Orientação Sociossexual (SOI) e as duas questões seguintes referentes ao questionário elaborado, demonstraram ambos que os homens se envolvem em práticas sexuais em maior proporção do que as mulheres e independente das circunstâncias, isto é, tanto em relações únicas e casuais quanto em duradouras (SCHMITT DP, 2005).

Como os aplicativos de relacionamento possibilitam a abrangência de parceiros, devido ao grande número de usuários inscritos em plataformas como o Tinder, por exemplo, foi possível confirmar que a predisposição biológica que distingue homens e mulheres durante a seleção de parceiros se manifesta também em aplicativos de relacionamento, já que mesmo que as mulheres possam aumentar o número de parceiros sexuais por meio do seu uso, se mantém restritas sexualmente, ao contrário dos homens, que preferem se envolver com uma variedade de mulheres (HATTORI WT e CASTRO FN, 2017; POLIPPO PM, et al., 2016).

Segundo Figueiredo LB (2016) é comum as mulheres sentirem um estranhamento e desconforto quando se envolvem em relacionamentos casuais estritamente sexuais, cuja razão não conseguem explicar. Isso 
ocorre, de acordo com Hattori WT e Castro FN (2017), em função das manifestações genéticas que as impulsionam para a emissão de determinados comportamentos que muitas vezes são reproduzidos sem sequer terem conhecimento e de modo inconsciente, com isso, não sabem explicar o porquê têm preferência por relacionamentos duradouros (BAILEY WC, et al., 1987; OVERBEEK G, et al., 2018).

Segundo Galperin A, et al. (2013) as mulheres se arrependem quando se envolviam em sexo casual, sem conseguirem argumentar o porquê se sentiam tristes após consumá-lo. Já os homens se lamentavam apenas quando consideravam que não obtiveram um desempenho sexual satisfatório. Kennair LEO, et al. (2018) obtiveram os mesmos dados referentes a repulsa feminina voltada à casualidade e verificaram uma expressão facial de nojo nas mulheres ao relatarem experiências de sexo casual vivenciadas no passado.

É importante ressaltar que a constituição humana e seu resultado final diz respeito tanto às contribuições genéticas como sociais. Desse modo, ao correlacionar os dados dessa pesquisa aos aspectos sociais que modulam o exercício sexual, é possível apontar o estudo de Chen AC, et al. (2016) que reforçam que às meninas são atribuídos valores culturais mais restritos e rigorosos em relação à manifestação da sexualidade e é incentivado socialmente que não se envolvam com muitos parceiros sexuais.

Sendo a amostra dessa pesquisa brasileira pode-se considerar os reflexos históricos provindos do machismo e conservadorismo sexual vivenciados no passado e aplicados principalmente à sexualidade feminina, em que aquelas que se envolviam em variadas práticas sexuais perdiam seu valor como esposa e o seu prestígio social (DANTAS BSA, 2010). Com isso, esses fatores quando são associados aos biológicos e genéticos contribuem ainda mais para a supressão sexual feminina. No entanto, Guerra VM, et al. (2012) mencionam que ter uma vivência sexual contribui para o liberalismo sexual do sujeito independentemente do gênero.

Segundo Serrano-Barquin C, et al. (2018), os homens obtiveram maiores vantagens sociais em relação à liberdade sexual e durante um período histórico a sexualidade feminina era atrelada unicamente ao prazer masculino e pouca atenção era dada as suas necessidades. Citam que a publicidade contribuiu para a propagação da ideia vinculada à mulher como objeto sexual dos homens e distinguiam aquelas consideradas para se casar, que correspondiam às que tiveram pouca ou nada experiência sexual e as que manifestam a sexualidade de modo explícito foram atribuídas características negativas.

Desse modo, é possível compreender que as punições sociais referentes ao exercício sexual feminino contribuem até hoje para a repressão sexual das mulheres e se reflete no seu baixo interesse sexual quando atrelada à predisposição biológica já existente, uma vez que estando inserida em uma sociedade constituída por normas e regras, corre o risco de ser julgada de modo pejorativo por seu parceiro virtual caso demonstre interesses sexuais.

Como mencionado acima, a usuária de aplicativos pode acreditar que será associada às relações estritamente casuais se aparentar estar dispostas a manter relação sexual e consequentemente perder seu valor como mulher considerada para se casar. Apesar da cultura patriarcal, em que a mulher era compreendida como submissa aos homens estar aos poucos sendo diluída e diversos direitos, como a liberdade sexual feminina, estarem sendo repensados, Dantas BSA (2010) menciona que ainda existem resquícios históricos e culturais dos comportamentos considerados corretos a serem seguidos e, dentre eles, o baixo envolvimento sexual feminino.

Entretanto, levando em consideração que os comportamentos não são imutáveis, Overbeek G, et al. (2018) apontam que os homens aumentaram o nível de restrição sexual quando tiveram pais conservadores, pois compartilharam os mesmos valores e crenças que aprenderam com eles, o que sugere que é possível modelar os comportamentos a partir de normas sociais.

Como os homens corresponderam ao grupo que está mais propenso a se envolver em relações sexuais com parceiros virtuais, estão mais expostos aos riscos referente às práticas sexuais e à contração de doenças sexualmente transmissível ou gravidez indesejada da parceira com quem se relacionou.

Já que não é possível modificar os fatores genéticos existentes na composição humana, Connell RW e Messerschmidt JW (2013) apontam ser possível realizar mudanças culturais que se atrelam aos hábitos e 
costumes e quando são reforçadas são capazes de proporcionar a modificação dos comportamentos indesejados e possibilitam a instalação de condutas desejáveis.

Portanto, como se sabe com os dados dessa pesquisa que os usuários de aplicativos de relacionamento estão abertos a terem relação sexual com os pretendentes virtuais no primeiro encontro, seja em maior ou menor propensão a depender do gênero, é necessário pontuar que a Educação Sexual seria um meio propício para se evitar um problema de saúde pública relacionado ao exercício sexual imprudente e negligente ao uso de contraceptivos. Com isso, reforçar a necessidade de exercer a sua sexualidade de maneira assertiva é necessário, uma vez que a prática sexual entre esse público foi confirmada.

A educação sexual apresentada ao sujeito desde a infância contribui para sua conscientização sexual e prevenções de quadros indesejáveis, segundo Furlanetto MF, et al. (2018). No entanto, os autores apontam que a Educação Sexual é tão falha no Brasil que se quer os professores responsáveis por transmitirem os conhecimentos, tem domínio sobre o assunto.

Ao invés dos educadores se basearem em estudos científicos para informar os alunos, partem de princípios e crenças religiosas, que contribuem para o aumento da imprudência sexual e falta de prevenção a partir da negligência do conhecimento, acesso e uso correto de preservativos.

Segundo Nascimento EGC, et al. (2017) o uso da camisinha deveria ser indispensável para a evitação de transmissões de doenças sexuais e gravidez não planejada, devendo ser costume de consumo tanto de homens quanto mulheres, no entanto, ressalta que para sua eficácia o sujeito deve utilizá-la de forma correta.

Aponta que apenas nas últimas duas décadas os estudos mundiais tem produzido literatura sobre o tema, em função do aumento da propagação da AIDS e anteriormente, as pesquisas em sua maioria, correlacionavam o uso do preservativo apenas à prevenção de gestação. As pesquisas brasileiras são ainda mais incipientes sobre o assunto e pouco discutem a intervenção preventiva relacionada à educação sexual e sexualidade de brasileiros.

Ainda que o foco do artigo tenha sido verificar a diferenças entre homens e mulheres a respeito do exercício sexual nos aplicativos de relacionamento e não abordou questões sobre o uso de preservativos, um estudo recente realizado por Moreira LR, et al. (2018) com estudantes universitários brasileiros, que configura uma amostra semelhante à da presente pesquisa e com faixas etárias parecidas, demonstrou que aproximadamente $60 \%$ deles não haviam utilizado nenhum tipo de preservativo na última relação sexual.

Nascimento EGC, et al. (2017) reforçam que de um modo geral, o uso específico da camisinha é negligenciado em brasileiros e sugerem que a educação sexual implementada e regulada pelo governo seria o meio mais eficaz para promover a adesão da população ao uso de preservativos.

Do mesmo modo, Sawyer NA, et al. (2018) e Green SM, et al. (2018) apontaram que os usuários dos aplicativos de relacionamento se envolvem em práticas sexuais mais perigosas do que os demais, abrangendo uma variedade de parceiros sem uso de preservativo e abuso de álcool e drogas ilícitas.

Arfer KB e Jone JJ (2018) e Guerra VM, et al. (2012) demonstraram que a modelação cultural é capaz de tornar o sujeito mais conservador sexualmente a partir da interiorização de valores políticos e sociais que norteiam o desejo de pertença ao grupo, isto é, o que se caracteriza como hábito social torna-se desejável para o sujeito. Overbeek G, et al. (2018) também identificaram que os valores que os pais repassam aos filhos influenciavam em sua dinâmica sexual e assim, os últimos se envolviam em mais comportamentos sexuais seguros, com proteção e houve aumento da restrição sexual masculina nesses casos.

O que se pretendeu ao mencionar os autores acima não foi propor uma cultura sexual conservadora no Brasil, mas sim explicitar dados que já demonstraram que as variáveis culturais possuem alto poder de impacto nas decisões sexuais dos sujeitos.

Com isso, o que se propõe é que a educação sexual seja inserida em variados ambientes para que seja possível modelar a cultura brasileira a partir de uma concepção de relação sexual segura e consciente e assim, independentemente do número de parceiros sexuais dos usuários dos aplicativos de relacionamento, eles estariam com sua saúde intacta devido à prevenção sexual. 


\section{CONCLUSÃO}

Foi possível concluir que os homens se envolvem em mais práticas sexuais com parceiras de aplicativos de relacionamento do que as mulheres e independente das circunstâncias, com isso, verificou-se que os fatores biológicos e culturais contribuem para tal irrestrição sexual masculina já que ambos estão presentes na constituição humana. A pesquisa foi relevante por contemplar as predisposições genéticas na escolha de parceiros amorosos, uma vez que as pesquisas brasileiras têm negligenciado esse viés e contemplado, na maioria das vezes, somente as influências culturais e sociais e por abordar o uso dos aplicativos de relacionamento, também pouco estudado até o momento em amostras brasileiras, tornou o estudo ainda mais relevante. Uma limitação foi não ter inserido uma questão a respeito do uso de preservativos entre os usuários dos aplicativos, já que esse dado poderia contribuir para uma maior compreensão dos relacionamentos virtuais.

\section{REFERÊNCIAS}

1. ARFER KB, JONE JJ. American political-party affiliation as a predictor of usage of an adultery website. Archives of Sexual Behavior, 2018; 1: 1-9.

2. BAILEY WC, et al. Relation of sex and gender role to in love, sexual attitudes, and self-esteem. Sex Roles, 1987; 16(12): 637-648.

3. BANDEIRA TTA, MOURA MLS. Crenças de pais e mães sobre investimento parental. Paidéia, 2012; 22(53): 355363.

4. CHEN AC, et al. Contextual influence of Taiwanese adolescents' sexual attitudes and behavioral intent. Nursing and Health Sciences, 2016; 18: 355-361.

5. CONNELL RW, MESSERSCHMIDT JW. Masculinidade hegemônica: repensando o conceito. Revista Estudos Feministas, 2012; 21(1): 241-282.

6. DANTAS BSA. Sexualidade, cristianismo e poder. Estudos e Pesquisas em Psicologia, 2010; 10(3): $700-728$.

7. FIGUEIREDO LB. Tinderellas: busca amorosa por meio de aplicativos para smartphone. 2016. Tese de Doutorado em Psicologia Clínica - Pontifícia Universidade Católica de São Paulo, São Paulo - SP.

8. FURLANETTO MF, et al. Educação sexual em escolas brasileiras: Revisão sistemática da literatura. Cadernos de Pesquisa, 2018; 48(168): 550-571.

9. GALPERIN A, et al. Sexual regret: Evidence for evolved sex differences. Archives of Sexual Behavior, 2013; 42: 11451161.

10. GREEN SM, et al. Exploring the Effect of Sharing Common Facebook Friends on the Sexual Risk Behaviors of Tinder Users. Cyberpsychology, Behavior, and Social Networking, 2018; 21(7): 457-462.

11. GUERRA VM, et al. Sexual liberalism-conservatism: the effect of human values, gender, and previous sexual experience. Archives of Sexual Behavior, 2012; 41: 1027-1039.

12. HATTORI WT, CASTRO FN. As origens do amor: evolução da escolha de parceiros. in VIEIRA ML, OLIVA AD, org. Evolução, Cultura e Comportamento Humano. Florianópolis: Edições do Bosque, 2017; 220-281p.

13. KENNAIR LEO, et al. Why do women regret casual sex more than men do?. Personality and Individual Differences, 2018; 127: 61-67.

14. MOREIRA LR, et al. Uso de preservativos na última relação sexual entre universitários: quantos usam e quem são?. Ciência e Saúde Coletiva, 2018; 23(4): 1255-1266.

15. NASCIMENTO EGC, et al. Adesão ao uso da camisinha: a realidade comportamental no interior do nordeste do brasil. Revista Salud Pública, 2017; 19(1): 39-44.

16. NOGUEIRA MFM, SILVA TT. O amor em suas mãos: um estudo sobre a sociabilidade entre os indivíduos no aplicativo tinder. Revista Científica de Comunicação Social, 2016; 6(2): 99-102.

17. OVERBEEK G, et al. Buffer or Brake? The role of sexuality-specific parenting in adolescents' sexualized media consumption and sexual development. Journal of Youth and Adolescence, 2018; 47: 1427-1439.

18. PINCOTT, J. Os homens preferem mesmo as loiras? A ciência por trás do sexo, do amor e da atração. Tradução de Angelo Ikeda. São Paulo: Editora Academia de Inteligência, 2010.

19. POLIPPO PM, et al. Produção científica brasileira sobre psicologia evolucionista. Gerais: Revista Interinstitucional de Psicologia, 2016; 9(2): 277-289.

20. RAMIREZ A, et al. When Online Dating Partners Meet Offline: The Effect of Modality Switching on Relational Communication Between Online Daters. Journal of Computer-Mediated Communication, 2014; 20: 99-114.

21. RAMOS CM, LENCASTRE MPA. O feminino e o masculino na etologia, sociobiologia e psicologia evolutiva: revisão de alguns conceitos. Psicologia, 2013; 27(2): 33-61.

22. ROCHA CTM, AMADOR FS. O teletrabalho: conceituação e questões para análise. Cadernos EBAPE.BR, 2018; 16(1): 152-162.

23. SANTAELLA L. A ecologia pluralista da comunicação: conectividade, mobilidade, ubiguidade. São Paulo: Paulus, 2010. 
24. SAWYER AN, et al. Dating Application Use and Sexual Risk Behavior Among Young Adults. Sexuality Research and Social Policy, 2018; 15(2): 183-191.

25. SERRANO-BARQUIN C, et al. Estereotipos de género que fomentan violencia simbólica: desnudez y cabellera. Revista Estudos Feministas, 2018, 3(26): 1-14.

26. SIMPSON JA, GANGESTAD SW. Individual differences in sociosexuality: evidence for convergent and discriminant validity. Journal of Personality and Social Psycology, 1991; 60(6): 870-883.

27. TRIVERS RL. Parental investment and sexual selection. In CHAMPBELL B, org. Sexual selection and the descent of man 1871-1971. Chicago: Aldine Publishing, 1972; 136-207p.

28. SÜMER ZHL. Effects of gender and sex-role orientation on sexual attitudes among turkish university students. Social Behavior and Personality, 2013; 41(6): 995-1008.

29. VERMELHO SC, et al. Sobre o conceito de redes sociais e seus pesquisadores. Educação e Pesquisa, 2015; 41(4): 863-881. 